\title{
INFECCIONES EN EL PIE DIABÉTICO
}

\section{Pérez Prieto}

Servicio de Cirugía Ortopédica y Traumatología. Hospital del Mar. Barcelona Unidad de Artroscopia Equilae. Hospital Universitario Quirón Dexeus. Barcelona

\section{Introducción y patogénesis}

La neuropatía periférica que sufren los pacientes con diabetes mellitus (DM) puede producir como consecuencia una extensa variedad de lesiones a nivel del pie y el tobillo. Entre las más frecuentes se encuentran las úlceras diabéticas, que en casos de evolución tórpida pueden requerir amputación hasta en un 15\% de los $\operatorname{casos}^{(1)}$. De hecho, padecer una infección de pie diabético (IPD) es la causa más frecuente de hospitalización de los pacientes con DM y la principal causa de amputación de extremidades inferiores ${ }^{(2)}$.

Las úlceras diabéticas se deben a múltiples factores, entre los que se encuentran la propia hiperglucemia mantenida y el aumento de la hemoglobina glicosilada (HbGlic), la arteriopatía y la neuropatía diabéticas ${ }^{(3-5)}$. No obstante, el riesgo de amputación mencionado se puede reducir hasta en un 50\% con educación sanitaria adecuada, manejo metabólico correcto de la DM, reducción de zonas de hiperpresión (de manera ortopédica o quirúrgica) y con la reducción de factores de riesgo cardiovascular ${ }^{(6)}$.

Por lo que respecta a las IPD, la posibilidad de padecerlas a lo largo de la vida en un paciente diagnosticado de DM es de hasta un 25\% ${ }^{(1)}$.

En ocasiones, las IPD se producen como celulitis (secundarias a heridas/erosiones superficiales), infecciones posquirúrgicas o postraumáticas. Sin embargo, lo más frecuente es que las IPD sean secundarias a las úlceras diabéticas comentadas con anterioridad.

La polineuropatía diabética conlleva una pérdida progresiva de sensibilidad proprioceptiva y protectora que puede conllevar la aparición de deformidades como el Charcot (véase el capítulo correspondiente) ${ }^{(7)}$, trastornos de la marcha y movilidad reducida que acarrea la aparición de zonas de hiperpresión que son muy susceptibles

FS $₫ 2018$ SEMCPT. Publicado por Imaidea Interactiva en FONDOSCIENCE ${ }^{\circledR}$ (www.fondoscience.com). 


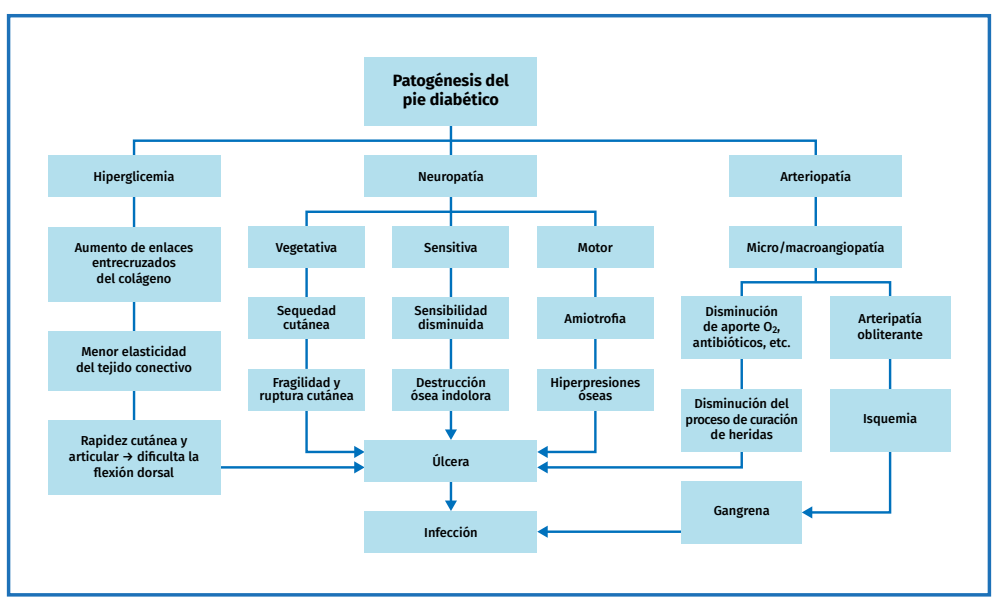

Figura 1. Patogénesis del pie diabético.

micas). Si bien es cierto que úlcera no es sinónimo de infección, la presencia de una úlcera diabética es una condición necesaria para la aparición de IPD ${ }^{(9)}$.

Las úlceras secundarias a vasculopatía diabética representan hasta un $37 \%$ de todos los casos. Las úlceras isquémicas aparecen en un pie frío y mal perfundido y son frecuentes en el borde lateral del 5.o metatarsiano y en zonas donde no existe hiperpresión. Además, se caracterizan por presentar escasa callosidad circundante. Es fundamental palpar los pulsos pedio y tibial posterior, así como la repleción vascular digital, y, en caso de

de sufrir úlceras diabéticas ${ }^{(2,8)}$. A ello se le une la insuficiencia arterial y trastornos inmunológicos debido al aumento de la HbGlic que disminuye la actividad de los leucocitos ${ }^{(4)}$. Todos estos factores imbricados son los que acaban produciendo una IPD (Figura 1).

\section{Úlceras diabéticas: tipos y clasificación}

En el paciente diabético pueden aparecer fundamentalmente 2 tipos de úlceras: las úlceras neuropáticas y las úlceras isquémicas. No obstante, en muchos casos pueden ser mixtas (neuroisqué-
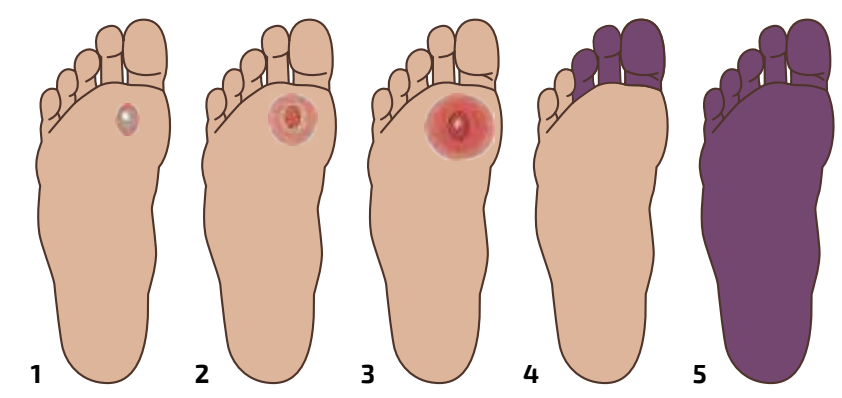

Grado 0: pie de riesgo, piel intacta.

Grado 1: úlcera superficial o que afecta tejido celular subcutáneo.

Grado 2: úlcera profunda que afecta tendón y/o capsula (sin osteomielitis).

Grado 3: úlcera profunda que afecta hueso (con osteomielitis).

Grado 4: gangrena que afecta únicamente dedos (amputación menor).

Grado 5: gangrena que afecta mediopié/antepié (amputación mayor).

Figura 1. Clasificación de Wagner para úlceras diabéticas. cualquier anomalía, realizar una eco-Doppler o indices vasculares digitales (el índice tobillo-brazo -ITB- puede ofrecer falsos negativos debido a calcificación vascular) $)^{(1,2)}$.

Las úlceras neuropáticas las padecen pacientes en los que la sensibilidad propioceptiva está disminuida. Ocurren en zonas de presión, típicamente en la planta del pie y también a nivel del talón en pacientes encamados. Es frecuente que aparezcan después de deformidades como la artropatía de Charcot, en la que la caída del arco plantar y la aparición de exóstosis crea zonas de hiperpresión previamente no presentes ${ }^{(7,10)}$. Típicamente, las úlceras neuropáticas presentan callosidad alrededor de las mismas.

La clasificación de Wagner es una de las más utilizadas para la clasificación de las úlceras diabéticas mediante una estadificación de 0 a 5 (Figura 2). La importancia de la presencia de isquemia y/o infección ha hecho que la Universidad de Texas haya añadido 5 posibles supuestos para cada estadio de Wagner(2):

- A: ni isquemia ni infección presentes.

- B: con infección asociada.

- C: con isquemia asociada.

- D: con isquemia e infección asociadas.

De esta manera, un estadio 2B sería un paciente con una úlcera en la que existe tejido tendinoso expuesto y además presenta infección. 


\section{Clínica y diagnóstico}

Cuando un paciente acude a consulta con sospecha de IPD, el pie debe ser minuciosamente examinado para describir las posibles lesiones cutáneas. No solo es importante describir la existencia o no de úlcera diabética, sino también la presencia de pequeñas erosiones (frecuentemente entre los dedos menores). También es importante anotar si existen deformidades o zonas de hiperpresión en riesgo, así como la presencia de pulsos periféricos.

Existen algunos signos clínicos que deben hacer sospechar IPD (1):

- La presencia de una úlcera que no evoluciona favorablemente a lo largo de 2 semanas a pesar de descartar vasculopatía y de realizar una descarga correcta es altamente sugestiva de infección.

- La presencia de edema, aumento de temperatura y eritema puede ser sugestiva tanto de infección como de Charcot en fase inflamatoria.

- Eritema de más de $2 \mathrm{~cm}$ alrededor de una úlcera crónica es sugestivo de infección grave.

- La presencia de un dedo engrosado y rojo (dedo en salchicha) es indicativo de osteomielitis.

- La existencia de secreción purulenta y mal olor son indicativos de IPD y además son signos de mal pronóstico.

Por lo que respecta a las pruebas de laboratorio, es imperativa la realización de una analítica general con reactantes de fase aguda (hemograma, velocidad de sedimentación globular -VSG-y proteína $C$ reactiva $-\mathrm{PCR}-$ ). No obstante, es muy importante recordar que la normalidad de los mismos no excluye la presencia de IPD, especialmente en las úlceras e infecciones crónicas ${ }^{(11)}$. Estos marcadores son especialmente útiles para monitorizar la respuesta al tratamiento(4).

La prueba probe to bone (palpar hueso en una úlcera con un palpador romo) es básica ya que, de acuerdo con el estudio de Shone, en caso de que se palpe hueso, la probabilidad de que exista osteomielitis es de un $87 \%^{(12)}$.

El diagnóstico por imagen es otro de los puntos clave. La primera prueba a solicitar es la radiología simple; en la mayoría de las ocasiones es suficiente con las proyecciones dorsoplantar y lateral de pies en carga. Se debe inspeccionar la imagen buscando osteolisis (estos signos osteomielíticos no aparecen antes de las 2 semanas), burbujas de aire (implicaría infección por anaerobios), deformidades o destrucción articular. Sin embargo, la prueba más fidedigna es la resonancia magnética (RM), con una sensibilidad de un $86 \%$ y una especificidad de un $92 \%$ para el diagnóstico de osteomielitis (aún más elevada para la presencia de abscesos). Por último, la tomografía computarizada (TC) puede ser necesaria para la planificación quirúrgica (destrucción ósea/articular, presencia de secuestro, etc.) $)^{(13)}$.

La parte quizás más importante en el diagnóstico es el cultivo microbiológico. Es crucial abandonar la práctica de realizar cultivos de tejido superficial, punción aspiración o cultivo mediante escobillones $^{(1,4)}$. Cualquiera de estas técnicas tiene una rentabilidad diagnóstica pésima, no solo por el elevado porcentaje de falsos negativos, sino también por la identificación de microorganismos saprófitos provenientes de la microbiota cutánea que no son causantes de infección. Es fundamental el envío de entre 3 y 5 muestras de tejido profundo para el estudio microbiológico. El mejor tejido es aquel que muestra signos de infección (friable, poco vascularizado, necrótico, etc.) y que circunda el hueso. Aunque el cultivo positivo de hueso es definitorio de osteomielitis, no es la mejor muestra a enviar, ya que el cultivo microbiológico es complejo y de bajo rendimiento. Existen técnicas como el vortexing o bead-mill que pueden optimizar el manejo del cultivo de muestras óseas. Por ello, es fundamental contar con un microbiólogo especialista en infecciones musculoesqueléticas ${ }^{(14)}$.

\section{Tratamiento de las infecciones del pie diabético}

Es importante volver a recordar que úlcera diabética no es sinónimo de infección. En aquellas úlceras de reciente aparición (menos de 2-3 semanas) y sin signos de infección (eritema, probeto-bone negativo, reactantes de fase aguda, etc.), el tratamiento debe ser eliminar las causas que han producido estas úlceras. El yeso de contacto total (YCT) ha demostrado ser el tratamiento de elección en los casos en los que es necesario descargar zonas de hiperpresión. Por lo que respecta al tratamiento local de la úlcera, es mandatorio mantenerla limpia y seca, ya sea mediante agentes mecánicos o químicos (ácido acético, 
clorhexidina, yodo, etc.) y, más recientemente, incluso agentes biológicos como los bacteriófagos. No obstante, ninguno de ellos ha demostrado superioridad sobre otro.

Además, en aquellos casos en los que exista un problema vascular que pueda impedir la curación de la úlcera, este debe ser resuelto. De hecho, las úlceras diabéticas deberían curar si se cumplen estos 3 supuestos:

- Existe un aporte vascular adecuado en la extremidad afecta.

- La infección se trata de manera adecuada si esta existe.

- Las áreas de hiperpresión se eliminan o descargan.

En caso de que exista una úlcera que no evoluciona favorablemente a pesar de realizar el tratamiento correcto y eliminar las causas que la generen, se debe plantear realizar un desbridamiento quirúrgico y la toma de muestras de tejido profundo para averiguar si existe una infección subyacente. El desbridamiento quirúrgico se debe plantear también en aquellos casos en que existan imágenes compatibles con osteomielitis o abscesos, en el paciente séptico con alteración de los reactantes de fase aguda o en aquellas úlceras que presenten signos de infección (drenaje purulento o eritema circundante). Aunque para muchos traumatólogos el desbridamiento se pueda considerar como una cirugía sencilla, es crucial concienciar de que debe ser un proceso meticuloso en el que se desbride y elimine todo tejido necrótico y poco viable. Se debe realizar tanto sobre los tejidos blandos como sobre el hueso, buscando el paprika sign, es decir, tejido

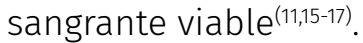

En aquellos casos en los que sea necesario eliminar gran parte de hueso necrótico/osteomielítico, será necesario el aporte de injertos o sustitutivos óseos, que deberán ser recubiertos o saturados en antibióticos para mejorar el resultado de control de la infección. Además, aquellos casos en que mecánicamente sea necesaria la fijación deberán ser evaluados para decidir si es factible la fijación interna, ya que proporciona mejores resultados en calidad de vida que la fijación externa. No obstante, estos casos son siempre complejos y deben ser evaluados y tratados por unidades que dispongan de un equipo multidisciplinar con microbiólogos, traumatólogos, infectólogos y cirujanos plásticos ${ }^{(15)}$.
Finalmente, por lo que respecta al tratamiento antibiótico, este siempre debe ser asociado a cirugía, ya que el tratamiento antibiótico aislado tiene una tasa de fallo de hasta el 30\%, favoreciendo la aparición de resistencias. Sin embargo, existen 2 supuestos en los que se podría plantear tratamiento antibiótico aislado (con estricto control evolutivo y planteando desbridamiento en caso de mala evolución):

- Celulitis sin colecciones.

- Osteomielitis aguda localizada.

El tratamiento empírico antibiótico inicial se debe realizar de manera sistémica. En la mayoría de los casos, la utilización de piperacilina/tazobactam endovenosa es adecuada hasta los resultados de los cultivos intraoperatorios. En aquellos casos en los que se sospeche infección por Staphylococcus aureus resistente a la meticilina (MARSA) (tratamientos antibióticos previos, pacientes institucionalizados), se debe valorar añadir vancomicina al tratamiento anterior. En cuanto al tratamiento oral, se debe buscar aquel que tenga una adecuada biodisponibilidad y aceptable penetración ósea. En este sentido, la combinación de ciprofloxacino a dosis altas $(750 \mathrm{mg}$ cada 12 h) y clindamicina (600 mg cada 8 h).

\section{Conclusiones}

La idea más importante de este capítulo es recordar que el tratamiento de las IPD son cuadros en los que es necesario contar con un equipo multidisciplinar especializado en el tratamiento de infecciones osteoarticulares. Finalmente, no menos importante es recordar la importancia de la prevención de esta patología.

\section{Bibliografía}

1. Ochsner PE, Borens O, Trampuz A, Uçkay I. Infektionen des Bewegungsapparates. Swiss Orthopedics; 2014.

2. Boulton AJ. The Diabetic Foot. En: De Groot LJ, Chrousos G, Dungan K, et al. (eds.). Endotext. South Dartmouth (MA): MDText.com, Inc.; 2000.

3. Crouzet J, Lavigne JP, Richard JL, Sotto A; Nîmes University Hospital Working Group on the Diabetic Foot (GP30). Diabetic foot infection: a critical review of recent randomized clinical trials on antibiotic therapy. Int J Infect Dis. 2011 Sep;15(9):e601-10. 
4. Lipsky BA, Berendt AR, Cornia PB, Pile JC, Peters EJ, Armstrong DG, et al. 2012 Infectious Diseases Society of America clinical practice guideline for the diagnosis and treatment of diabetic foot infections. Clin Infect Dis. 2012 Jun;54(12):e132-73.

5. Uçkay I, Aragón-Sánchez J, Lew D, Lipsky BA. Diabetic foot infections: what have we learned in the last 30 years? Int J Infect Dis. 2015 Nov;40:81-91.

6. Ndosi M, Wright-Hughes A, Brown S, Backhouse M, Lipsky BA, Bhogal $M$, et al. Prognosis of the infected diabetic foot ulcer: a 12-month prospective observational study. Diabet Med. 2018 Jan;35(1):78-88.

7. Pinzur MS, Sammarco VJ, Wukich DK. Charcot foot: a surgical algorithm. Instr Course Lect. 2012;61:423-38.

8. Roy KJ. Force, pressure, and motion measurements in the foot: current concepts. Clin Podiatr Med Surg. 1988 Jul;5(3):491-508.

9. Abbas M, Uçkay I, Lipsky BA. In diabetic foot infections antibiotics are to treat infection, not to heal wounds. Expert Opin Pharmacother. 2015 Apr;16(6):821-32.

10. Mutluoglu M, Lipsky BA. Diabetic foot osteomyelitis. CMAJ. 2016 Dec 6;188(17-18):E535.

11. Anakwenze OA, Milby AH, Gans I, Stern JJ, Levin LS, Wapner KL. Foot and ankle infections: diagnosis and management. J Am Acad Orthop Surg. 2012 Nov;20(11): 684-93.
12. Shone A, Burnside J, Chipchase S, Game F, Jeffcoate W. Probing the validity of the probe-to-bone test in the diagnosis of osteomyelitis of the foot in diabetes. Diabetes Care. 2006 Apr;29(4):945.

13. Lauri C, Tamminga M, Glaudemans AWJM, Juárez Orozco LE, Erba PA, Jutte PC, et al. Detection of Osteomyelitis in the Diabetic Foot by Imaging Techniques: A Systematic Review and Meta-analysis Comparing MRI, White Blood Cell Scintigraphy, and FDG-PET. Diabetes Care. 2017 Aug;40(8):1111-20.

14. Corvec S, Portillo ME, Pasticci BM, Borens O, Trampuz A. Epidemiology and new developments in the diagnosis of prosthetic joint infection. Int J Artif Organs. 2012 Oct;35(10):923-34.

15. Pérez-Prieto D, Portillo ME, González-Lucena G, Ginés-Cespedosa A. Foot and ankle infections: debridement, early fixation and rifampicin provide earlier recovery of function and quality of life. Foot Ankle Surg. 2017. [Epub ahead of print].

16. Malizos KN, Gougoulias NE, Dailiana ZH, Varitimidis S, Bargiotas KA, Paridis D. Ankle and foot osteomyelitis: treatment protocol and clinical results. Injury. 2010 Mar;41(3):285-93.

17. Donley BG, Philbin T, Tomford JW, Sferra JJ. Foot and ankle infections after surgery. Clin Orthop Relat Res. 2001 Oct;(391):162-70. 